\title{
Hierarchically porous polymer derived ceramics: A promising platform for multidrug delivery systems
}

\author{
Cekdar Vakifahmetoglu ${ }^{\text {a,* }}$, Damla Zeydanli ${ }^{\mathrm{b}}$, Veli Cengiz Ozalp ${ }^{\mathrm{c}}$, Barış Ata Borsa ${ }^{\mathrm{d}}$, Gian Domenico Soraru ${ }^{\mathrm{e}}$ \\ a Department of Materials Science and Engineering, Izmir Institute of Technology, 35430 Urla, Izmir, Turkey \\ ${ }^{\mathrm{b}}$ Department of Chemistry, Istanbul Technical University, 34469 Istanbul, Turkey \\ ' Department of Bioengineering, Konya Food \& Agriculture University, 42080 Konya, Turkey \\ d Department of Medical Microbiology, Faculty of Medicine, Yeditepe University, 34755 Istanbul, Turkey \\ e Department of Industrial Engineering, University of Trento, Via Sommarive 9, 38123 Trento, Italy
}

\section{H I G H L I G H T S}

- SiOC ceramics were fabricated by a simple technique to obtain high surface area (reaching $774 \mathrm{~m}^{2} / \mathrm{g}$ ) hierarchical porosity.

- Porous SiOC material were found to be biocompatible with less hemolysis than MCM-41.

- The loading capacity and the release kinetics of cargo loaded SiOC were better than that of MCM-41.

- It was demonstrated that hierarchical porosity ceramic can be used to carry different sized drug molecules.

- The antimicrobial activity tests show that SiOC performs better than MCM41 in improving bactericidal activity.

\section{A R T I C L E I N F O}

\section{Article history:}

Received 23 August 2017

Received in revised form 20 November 2017

Accepted 21 November 2017

Available online 23 November 2017

\section{Keywords:}

Hierarchical porous ceramics

High surface area

Multi drug (cargo) delivery/release system

\section{G R A P H I C A L A B S T R A C T}

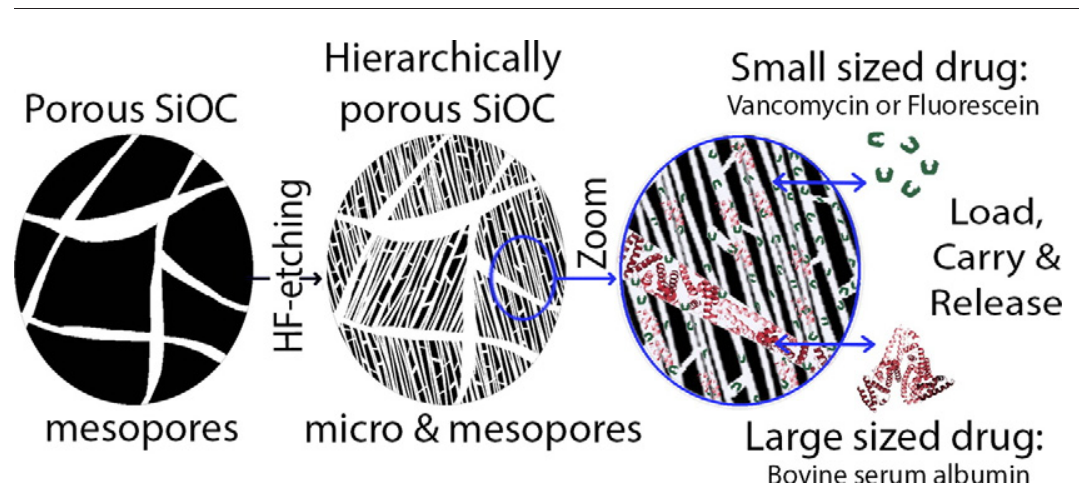

\begin{abstract}
A B S T R A C T
Mesoporous silicon oxycarbide (SiOC) components were formed with the use of "molecular spacer" (a sacrificial vinyl-terminated linear siloxane which while decomposing during pyrolysis generates pores with size proportional to the molecular weight), followed by a post-pyrolysis etching treatment by hydrofluoric acid (HF) to obtain C-rich SiOC samples having additional micro-/mesoporosity and specific surface area reaching to $774 \mathrm{~m}^{2} / \mathrm{g}$. The biocompatibility of the samples was validated by hemolysis test, and their cargo/drug loading capacities were studied by two different sized polypeptides as model molecules. SiOC particles showed less hemolysis compared to the reference material MCM-41. Similarly, the loading capacity and the release kinetics of bovine serum albumin (BSA) and vancomycin-loaded SiOC particles were improved compared to that of MCM-41. In the multi cargo loading/release capacity tests, done by using different sized molecules, Bio2-HF and MCM- 41 were loaded both with fluorescein and BSA. While a lagging time in fluorescein release was observed for MCM-41, the release kinetics of fluorescein and BSA was not affected when they are loaded together in the hierarchical pores of Bio2$\mathrm{HF}$, allowing the release of both large and small cargo molecules. The antimicrobial activity tests showed that Bio2-HF performed better than MCM-41 particles in improving bactericidal activity.
\end{abstract}

(c) 2017 Elsevier Ltd. All rights reserved.

\footnotetext{
* Corresponding author.

E-mail addresses: cekdarvakifahmetoglu@iyte.edu.tr, cvahmetoglu@gmail.com (C. Vakifahmetoglu).
} 


\section{Introduction}

Numerous processing routes have been explored for the production of porous ceramics, and several reviews are already available on the subject [1-5]. In order for a porous component to possess a wide range of characteristics, such as rapid transport, high selectivity, fast uptake and release, etc. it should comprise an interconnected cellular framework of different pore scales, i.e. hierarchical porosity [3]. Different types of hierarchical porosity may exist, according to the range of pore sizes that are involved in the porous structure, i.e. bimodal size distribution (micro-meso, meso-macro, micro-macro), or trimodal (micromeso-macro). The presence of "small" pores (particularly micropores, pores $<2 \mathrm{~nm}$ ) provides a high SSA (specific surface area) i.e. geometric area per unit mass of the part making the material functioning for a given application while macropores (pores $>50 \mathrm{~nm}$ ) offer mechanical stability as well as high transfer rate.

Hierarchical porosity components have also been investigated quite extensively [6-16]. Those studies have shown that the use of preceramic polymers offers advantages to create such porous structures since it is possible to use a wide range of methods borrowed either from low-cost plastic forming techniques such as extrusion, melt spinning, injection molding, blowing, etc. or from advanced ones such as additive manufacturing, electrospinning or aerogel processing [3,17-20].

Biocompatible material performs a medical therapeutic function without undesirable local or systemic effects in the patient [21]. Drug delivery systems are biocompatible carriers used for the targeted delivery/controlled release of therapeutic drugs [22,23]. For example, sitespecific and rate-controlled release of antibiotics can be innovative delivery systems to combat the increasing antibiotic resistance development problem. Accordingly, controlled release of antibiotics such as vancomycin, a glycopeptide antibiotic that is used to treat infections caused by Gram positive bacteria, has been explored to increase drug efficacy and patient compliance.

Among the drug delivery systems developed, mesoporous silica nanoparticles (MSN), especially MCM- 41 and SBA- 15 , have been extensively exploited for controlled drug release systems [23,24]. These high surface area mesoporous materials, generally speaking, are constituted by monomodal porosity which could be tailored to some extend in the mesopore range (2-50 $\mathrm{nm}$ ) [25]. Yet hierarchically architectured porous silica particles, seen in the nature, have rarely been used for such purpose [26]. Various drug molecules have been hosted by MSN particles synthesized with several different methods [23]. Apart from MSN, other inorganic porous materials such as zirconia, alumina, titania, etc. have also been produced for potential drug delivery systems [27]. In a recent study, Tamayo et al. [28] prepared a bimodal mesopore size distribution polymer derived ceramic (PDC); silicon oxycarbide (SiOC), functionalized with amino groups, and studied the release kinetics of Tenofovir cargo. However, despite extensive research on preceramic polymers and related PDCs, these materials were seldom tested in other medical applications [29-31]. Silicon oxycarbide is an anionic modification of silica glass in which part of the divalent oxygen atoms are substituted by tetravalent carbon atoms. This structural modification results into improved mechanical, chemical and thermal properties compared to the parent $\mathrm{SiO}_{2}$ glass [32].

Aforementioned studies have typically attempted to develop a carrier system to load a single drug, however loading a single cargo may not suffice to achieve ideal therapeutic efficacy, as in the case for chemotherapeutic drugs. As known, for the treatment of metastatic cancers a resistance to single agent occurs, and it can be overcome by combination chemotherapy using multiple anticancer agents producing a synergetic effect [33]. While dual/multi cargo carrier related works recently have been reported in the literature [33-35], such works used mesoporous particles (e.g. MSN). Similarly, albeit hierarchical materials exhibited promising bio-properties, to the best of our knowledge, there is no other study that focused on the deliberate formation of hierarchical porosity materials such as the ones proposed in this study, i.e. C-rich SiOC, and tested them with two very different sized cargo molecules for dual cargo delivery applications.

In the present work, following a relatively simple synthesis route, pristine SiOC samples designed to be mesoporous or just being nonporous have been obtained. Silica regions of these samples were further etched by hydrofluoric acid (HF) to obtain C-rich SiOC comprising additional micro-/mesoporosity and high SSA. These hierarchically porous SiOC samples were studied to demonstrate that a material with hierarchical porosity can be a promising platform to carry multiple drugs (cargo molecules) simultaneously, i.e. as an alternative drug delivery material which can carry and release cargo combination simultaneously. The samples were investigated for hemocompatibility, loading capacity with two different sized polypeptides (vancomycin or fluorescein and bovine serum albumin, BSA) as model cargo (drug) molecules, and antimicrobial activity. Commercially available mesoporous silica (MCM-41) was also characterized as reference material [25].

\section{Experimental procedure}

\subsection{Materials}

Mesoporous silica (MCM-41) was purchased from Sigma (CAS\# 763186-9, Sigma-Aldrich, Munich, Germany). Polymethylhydrosiloxane (Gelest, PHMS, $\mathrm{M}_{\mathrm{W}} \sim 2100-2400,30-45$ cST, CAS\# 63148-57-2, Gelest, Morrisville, PA, USA) having Si-H bonds, vinyl-terminated polydimethylsiloxane (PDMS, MW:62700, 10000 cSt, CAS\# 68083-19-2, Gelest, Morrisville, PA, USA) and a cyclic 2,4,6,8-tetramethyl-2,4,6,8tetravinlycyclotetrasiloxane (TMTVS 97\%, Alfa Aesar, Ward Hill, MA, USA, CAS\# 2554-06-5) with $\mathrm{Si}-\mathrm{C}=\mathrm{C}$ moieties, were used as preceramic precursors together with platinium - divinyltetramethyldisiloxane complex, Pt 2\% in xylene (CAS\# 68478-92-2, Sigma-Aldrich, St. Louis, MO, USA) serving as catalyst for the curing reactions. Hydrotalcite, (LDH with a chemical formula of $\mathrm{Mg}_{6} \mathrm{Al}_{2}\left(\mathrm{CO}_{3}\right)(\mathrm{OH})_{16} \cdot 4 \mathrm{H}_{2} \mathrm{O}$, CAS\# 11097-599, Sigma-Aldrich, St. Louis, MO, USA), was used as received.

PHMS/LDH/PDMS/TMTVS when 1/0.055/0.25/1 the sample coded as Bio1, and when 1/0.055/1/1 the sample coded as Bio2. The chemicals (all in mass ratio) were put into a glass beaker and mixed under magnetic stirring at room temperature (RT) for $5 \mathrm{~min}$ at $500 \mathrm{rpm}$ until the homogeneous mixture was achieved. Upon homogenization, 100 ppm by weight of Pt relative to PHMS was added into the mixture in a drop-wise manner. The blend was further mixed at $500 \mathrm{rpm}$ for $25 \mathrm{~min}$, and transferred into aluminum molds standing on the hot plate operating at $200{ }^{\circ} \mathrm{C}$ for curing overnight, followed by pyrolysis in an alumina tubular furnace (PROTERM PTF 16/75/450, Ankara, Turkey) at $1300{ }^{\circ} \mathrm{C}$ with a heating rate of $2{ }^{\circ} \mathrm{C} / \mathrm{min}$ and $1 \mathrm{~h}$ dwell time at the maximum temperature under $\mathrm{N}_{2}$ gas flow $(100 \mathrm{ml} / \mathrm{min})$. The etching process was conducted on ca $250 \mathrm{mg}$ of SiOC bulk sample using $50 \mathrm{ml}$ of $\mathrm{HF}$ ( $48 \mathrm{vol} \%$ in $\mathrm{H}_{2} \mathrm{O}$ ) solution in PE closed containers [36]. The attack was performed by leaving the sample in contact with the etching solution for 4 days at RT and gently stirring the containers by hand time to time. After the attack, SiOC samples were filtered, rinsed with distilled water (several times) and dried at $65^{\circ} \mathrm{C}$ for $24 \mathrm{~h}$ for the subsequent analysis.

\subsection{Characterization}

The morphological features were analyzed from fresh fracture surfaces using a Scanning Electron Microscope (SEM, FEI-Philips XL30 ESEM-FEG) after $10 \mathrm{~nm}$ Pt film deposition by sputtering. For Transmission Electron Microscopy (TEM, Philips CM12 (The Netherlands), tungsten thermionic source, $120 \mathrm{kV}$ accelerating voltage) the samples were ground in an agate mortar and dispersed in ethanol for $5 \mathrm{~min}$. A drop of solution was placed onto a carbon coated copper grid, followed by drying and analysis. X-ray diffraction data (XRD, Rigaku, DB-max) were collected using $\mathrm{Cu} \mathrm{K}_{\alpha 1}$ radiation ( $40 \mathrm{kV}, 30 \mathrm{~mA}$, step scan of $0.05^{\circ}$, counting time of $3 \mathrm{~s} / \mathrm{step}$ ). Both the un-etched and etched 
powders were analyzed by Nitrogen $\left(\mathrm{N}_{2}\right)$ gas adsorption. The isotherms were collected at $77 \mathrm{~K}$ using an ASAP 2010 (Micromeritics, Norcross, GA, USA) after degassing the sample at $200{ }^{\circ} \mathrm{C}$ for minimum $4 \mathrm{~h}$ before analysis. SSA was calculated from a BET (Brunauer, Emmet, and Teller) analysis in the $P / P_{0}$ range of $0.05-0.30$ using a minimum of five data points. The pore size distributions (PSDs) were obtained from the desorption branch of the isotherm through the BJH (Barret, Joyner, and Halenda) analysis.

\subsection{Hemolysis}

Whole human blood was collected and used within $8 \mathrm{~h}$. About $1 \mathrm{ml}$ of whole blood was added to $8 \mathrm{ml}$ of Dulbecco's phosphate buffered saline (D-PBS) and the Red Blood Cells (RBCs) were isolated from serum by centrifugation at $10,000 \times \mathrm{g}$ for $1 \mathrm{~min}$. The RBCs were washed two times with D-PBS solution. Finally, the RBCs were diluted to $1 \mathrm{ml}$ of DPBS. For each experiment, $50 \mu \mathrm{l}$ of the diluted RBC suspension was added to $50 \mu \mathrm{l}$ of silica or SiOC particle suspension in D-PBS at the concentration of $1.0 \mathrm{mg} / \mathrm{ml}$ and incubated for $4 \mathrm{~h}$ with shaking. After that, the mixture was centrifuged and the supernatants were assayed for hemoglobin content by Drabkin's method [37] by measuring the absorbance value at $577 \mathrm{~nm}$ with the reference wavelength at $655 \mathrm{~nm}$. All samples were prepared in triplicate. The percent of hemolysis was calculated as: (Hemolysis \% $=[($ Sample absorbance - negative control $) /$ (positive control - negative control)] $\times 100 \%$ ).

\subsection{Time-kill curves}

The bacterial susceptibility was studied by determining minimal inhibitory concentrations (MICs) in 96-well microtiter plates using the broth microdilution method as described in the clinical and laboratory standards institute [38]. Mueller Hinton Broth (MHB) was used for susceptibility testing as it is the common medium used for Staphylococcal cultures [39].

\subsection{Loading \& release}

SiOC or MCM-41 particles were loaded with BSA and/or vancomycin by incubating in $2 \mathrm{mg} / \mathrm{ml} \mathrm{BSA}$ and $100 \mu \mathrm{M}$ vancomycin in PBS overnight. The mixture was then washed with PBS before it is used in the experiments. Release of vancomycin from pores was monitored in a UV/VIS spectrophotometer (Agilent, 8000, Santa Clara, USA) at $280 \mathrm{~nm}$ as described [39]. BSA release was monitored by Bradford protein assay [40]. The particles were incubated for the required time in PBS buffer and the supernatants obtained after centrifugation at $12000 \mathrm{rpm}$ for $1 \mathrm{~min}$ were assayed for vancomycin or BSA. The solution was stirred continuously to create a homogeneous solution during incubation. The maximum loading of the particles with vancomycin or BSA was determined by preparing a mixture of $1 \mathrm{mg}$ particle mixture in PBS buffer and measuring the $280 \mathrm{~nm}$ absorbance in a well-mixed solution.

\section{Results and discussion}

\subsection{Morphological characterization}

Small sized $(<1 \mathrm{~mm})$ pieces were used in HF etching and following cargo release tests. In Fig. 1 (a-d) such pieces obtained from the sample, before (Bio1) and after HF (Bio1-HF) etching, are shown. While at the lower magnification (Fig. 1 (a \& b)) similar structures can be observed, comparing the higher magnification images given in Fig. 1(c) and (d) taken from the samples before and after etching respectively, the effect of acid treatment can evidently be seen. The roughness increases consistently with the formation of nano-sized pores upon acid treatment.
Fig. 2(a), (b) and (c) shows the TEM image of MCM-41, Bio1-HF and Bio2-HF, respectively. The differences in pore structures can evidently be seen. MCM-41 is constituted by well-defined monomodal mesopores below $5 \mathrm{~nm}$ in diameter (see later for $\mathrm{N}_{2}$ ads./des. data), instead in $\mathrm{HF}$ treated SiOC samples, there are different sized mesopores (from 2 to $50 \mathrm{~nm}$ ) together with macropores bigger than $50 \mathrm{~nm}$. Besides, sample Bio2-HF demonstrates higher number of large sized mesopores compared to Bio1-HF. Concerning the applications, such type of porosity character is important since it is known that macroporous framework provides high external mass transfer rate due to interconnections between the channels, and low pressure drop, while the micro/mesoporous system provide the functionality (bio-for the present case) for a given application.

\subsection{Structural characterization}

\subsection{Porosity characterization}

We have synergistically combined two approaches to prepare porous SiOC bodies: (i) utilization of PDMS (vinyl-terminated) for the purpose of "molecular spacer" to promote the formation of pores in the 10-20 $\mathrm{nm}$ range after pyrolysis [41], and (ii) a post-pyrolysis HF etching to dissolve the silica clusters present in the SiOC microstructure $[5,42$, 43] forming pores below $5 \mathrm{~nm}$ in size. The vinyl-terminated linear PDMS bonds with the pre-ceramic polymer (PHMS) and acts as a "molecular spacer" pushing apart the PHMS chains. During pyrolysis the PDMS decomposes completely creating pores whose size is related to the PDMS molecular weight [41]. It is known from the literature that the MW of the PDMS used in this study should lead to the formation of pores in the range $10-30 \mathrm{~nm}$. Indeed, as could be seen from the Table 1, with increasing the PDMS amount, the SSA values increased even without HF treatment. When the sample was made by using 0.25 parts of PDMS (i.e. Bio1), the resulting SSA was only $4.3 \mathrm{~m}^{2} / \mathrm{g}$ but quadrupling PDMS in the blends (Bio2, i.e. 1 part PDMS with 1 parts PHMS) the SSA of $121.9 \mathrm{~m}^{2} / \mathrm{g}$ was obtained, similar to previous work [44]. Comparable SSA increase due to additionally formed mesoporosity was also observed in previous studies $[41,45]$.

The $\mathrm{N}_{2}$ adsorption-desorption isotherms (not shown for brevity) recorded on the reference MCM-41 sample show the typical shape of mesoporous materials with the hysteresis loop close to $\mathrm{P} / \mathrm{P}_{0}=0.4$ suggesting the presence of pores of $\sim 3 \mathrm{~nm}$ size, in agreement with the published literature $[46,47]$. The BET surface area and total porosity values measured for the MCM-41 silica sample are $744 \mathrm{~m}^{2} / \mathrm{g}$ and $0.914 \mathrm{~cm}^{3} / \mathrm{g}$ as also reported in Table 1.

The sorption isotherms recorded on the samples before and after HF attacks are reported in Fig. 3(a). While sample Bio1 shows almost flat sorption isotherm typycal of low porosity material with the increase in PDMS amount, a clear hystherisis loop appears above $0.8 \mathrm{P} / \mathrm{P}_{0}$ indicating the presence of meso-macroporosity in the sample, see Fig. 3(a). After HF etching all these isotherms change, showing a steep increase at $\mathrm{P} / \mathrm{P}_{0}<0.1$ identifying microporosity which additionally formed during acid treatment. Indeed it is demonstrated that silicon oxycarbide have a complex nanostructure with a $\mathrm{SiO}_{2}$-rich and a C-rich interpenetrating nano-sized networks $[48,49]$. The silica rich "phase" can be etched out with a post-pyrolysis treatment with HF leaving behind a porous network whose typical size is a function of the maximum pyrolysis temperature and time [50].

In Fig. 3(b) pore volume vs. pore size curves are given. While the sample with low amount of PDMS (Bio1) shows almost no mesomacroporosity measured by $\mathrm{N}_{2}$ sorption test, increasing the PDMS fraction (Bio2) resulted in the formation of mesopores in the 10-20 nm size range $[41,44]$. After HF etching, samples showed three-modal pores, namely: micropores below $2 \mathrm{~nm}$, pores having 4-5 nm similar to previously work [51], and while the intensity is not as high as before, pores with sizes larger than $5 \mathrm{~nm}$ up to $\sim 50 \mathrm{~nm}$, i.e. hierarchical porosity, corraborating TEM data. 

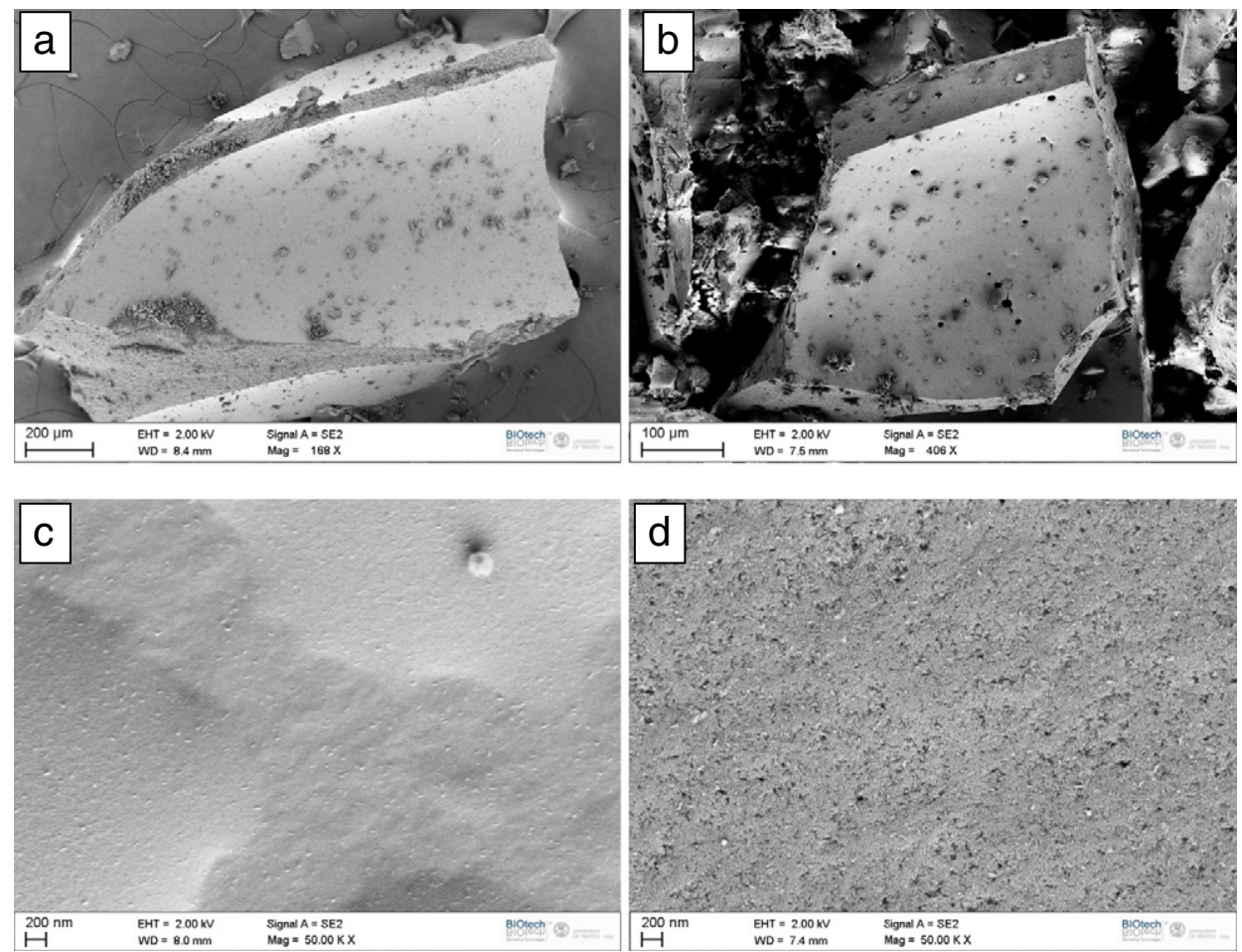

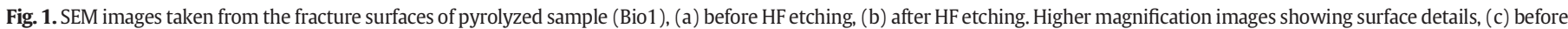
HF etching, and (d) after HF etching.

\subsection{Biomaterial properties: Hemolysis}

Most of the biomedical applications of nanoparticles involve a direct contact with blood. Thus, hemocompatibility studies have frequently been investigated to understand blood-compatible behavior of the potential application. The hemolytic activity of samples was determined by spectroscopic assay to measure hemoglobin amount, which is a direct indication of the integrity of RBCs. Although we used MCM-41 particles for comparison purposes, they are not similar in size or shape. However, they are the most suitable particles to be compared for hemolysis properties. As shown in Fig. 4(a), the hemolytic properties of SiOC particles were lower than that of the silica (MCM-41). Bio1 resulted in the least hemolytic activity among all the studied SiOC particles, which was $31 \%$ of the value measured for the MCM-41. Fig. 4(a) shows that HF etching treatment resulted in an increase in the hemolytic activity of SiOC particles, yet less hemolytic than MCM41 particles. Bio2-HF was the most hemocompatible among HF etched samples with a hemolytic activity of $18 \%$ less compared to MCM-41. It is highly probable that these results are related with two factors; (i) specific surface area, and (ii) surface silanol density [52]. The increase in surface area, enhances the contact with RBCs therefore may rise the hemolytic activity, but more pronounced effect comes from the latter. For all SiOC system investigated (both for etched and not-etched), there were fewer "accessible" Si-OH groups, i.e. the surface density of silanol groups were lower in SiOC compared to MCM-41. It should be underlined that HF treatment creates additional micro-mesoporosity
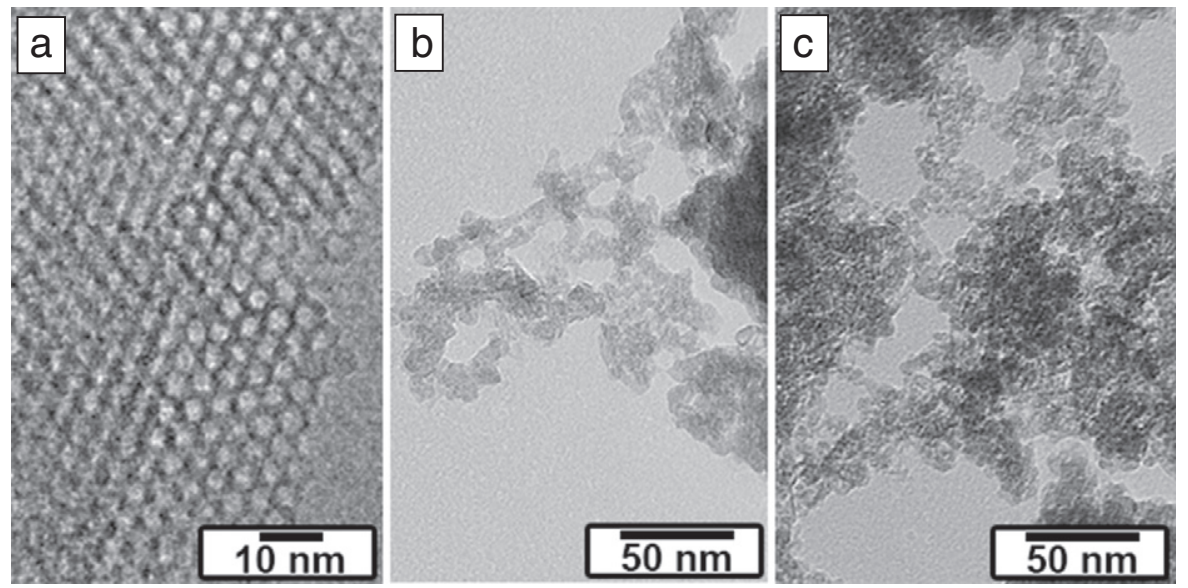

Fig. 2. TEM images taken from (a) MCM-41, (b) Bio1-HF, and (c) Bio2-HF. 
Table 1

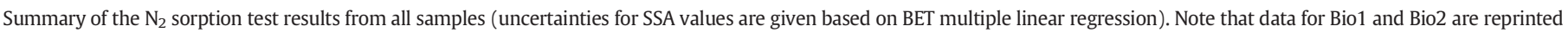
with permission from ref. [44].

\begin{tabular}{|c|c|c|c|}
\hline & $\operatorname{BET}\left(\mathrm{m}^{2} / \mathrm{g}\right)$ & Micropore volume $\left(\mathrm{cm}^{3} / \mathrm{g}\right)$ & Pore volume $\left(\mathrm{cm}^{3} / \mathrm{g}\right)$ - BJH desorption cumulative volume of pores \\
\hline Bio1 & $4.3 \pm 0.03$ & 0.001 & 0.023 \\
\hline Bio2 & $121.9 \pm 0.2$ & 0.004 & 0.473 \\
\hline Bio1-HF & $774 \pm 13$ & 0.363 & 0.441 \\
\hline Bio2-HF & $663 \pm 7.5$ & 0.139 & 0.581 \\
\hline MCM-41 & $744 \pm 10$ & 0.161 & 0.914 \\
\hline
\end{tabular}

via removal of "silica"-rich regions, and so new surfaces richer in carbon are being created $[20,53]$. Such process is actually a simple alternative way of surface functionalization while tailoring surface area, surface energy and nanorugosity index; it eventually enhances the interaction between the cargo molecule (i.e. drugs) and host surface.

The loading capacity of the particles is another important parameter in medical applications. In this study, two polypeptides with different size were employed as model cargo molecule to study the effects of hierarchical porosity properties. Bovine Serum Albumin (BSA) is the major blood serum protein, frequently employed as model peptide in medical research. With $1.4 \times 4.0 \times 4.0 \mathrm{~nm}^{3}$ dimensions, it consists of 589 amino acids making a molecular weight (MW) of 66,463 Da. Vancomycin is a glycopeptide has antibiotic properties and it is about 20 times smaller in volume than BSA with dimensions of $0.8 \times 1.5 \times 1.0 \mathrm{~nm}^{3}$ (MW of $1449.3 \mathrm{Da}$ ) [54].

The results in Fig. 3(b) showed that Bio2-HF particles demonstrated higher loading capacity, compared to particles with only mesopores (MCM-41). The hierarchical SiOC particles seem to be suitable for medical application with higher loading capacity both for small peptide, vancomycin and for a large protein, BSA. $1 \mathrm{mg}$ of Bio1 could adsorb 13 pmol of BSA, and HF-etching increased the adsorption capacity to 49 pmol. With a higher capacity, one mg of Bio2 particle adsorbed 31 pmol BSA, and after HF-etching 180 pmol BSA was loaded. Instead, mesoporous silica control sample MCM-41 adsorbed 47 pmol BSA. In all samples tested, vancomycin adsorption was at lower levels compared to BSA molecules, which can be explained by different interaction opportunities with the surface, resulting from more contact sites in BSA $[55,56]$. Bio 1 adsorbed 4 and 8 pmol vancomycin, respectively before and after HF-etching. Similarly, HF-etching increased vancomycin loading capacity of Bio2-HF about two times from 8 to 19 pmol while MCM41 particles could adsorb 14 pmol. In summary, Bio2-HF adsorbed about 3.8 times more BSA and about 1.4 times more vancomycin molecules than that of MCM-41. The reason behind such an observation lies in: (i) surface chemistry, which seems not suffice to explain the differences in the adsorption capacity of the Bio1-HF and Bio2-HF since surface chemistry of these etched samples should be quite similar, and (ii) differences in pore characteristics, the pore volume of Bio2-HF is much higher than that of Bio1-HF, besides the majority of the porosity for Bio2-HF localized in the 3-10 nm range which seems to be a very functional pore architecture to adsorb such molecules, yet for Bio1-HF, the porosity is spread over a wide range, see Fig. 3(b). Note also that MCM-41 has monomodal porosity around $\sim 3 \mathrm{~nm}$.

\subsection{Drug release}

BSA is a globular protein that is commonly used in the biochemical applications. Bradford protein determination assay is based on absorption maximum of Coomassie Brilliant Blue associated with peptide bonds. The BSA concentrations in release experiments were determined by comparing to standard BSA concentrations. Vancomycin is a short peptide, consisting of two kinds of amino acids, namely Alanine and Lysine. Peptide bond absorbs strongly in the far UV region with an absorption band at $280 \mathrm{~nm}$ that can be used to calculate molar extinction coefficient according to Beers-Lambert law. The vancomycin concentrations in release experiments were determined by comparing to standard vancomycin concentrations. Cargo loading values were already supplied in Fig. 4(b).

Cargo release properties of samples were first tested in vitro by monitoring BSA or vancomycin release by UV absorbance method. The particles loaded with either vancomycin or BSA was incubated in PBS buffer. The cumulative release of cargo increased up to $5 \mathrm{~h}$ and leveled off afterwards. MCM-41 particles released about $78 \%$ of loaded BSA in $4.5 \mathrm{~h}$ and $80 \%$ at the end of one day incubation (Fig. 5(a)). Bio2 particles demonstrated a similar release profile with $78 \%$ release in $4.5 \mathrm{~h}$ while Bio1 particles showed slightly slower release rate with $65 \%$ release in $4.5 \mathrm{~h}$ (Fig. 5(a)). However, for the etched samples, the release amount increased, for Bio2-HF to $86 \%$ for initial 4.5 h and $90 \%$ for one-day incubation.

The amount of vancomycin release from MCM-41 reached to $78 \%$ in $4.5 \mathrm{~h}$, which is in agreement with previous reports [57], and analogous
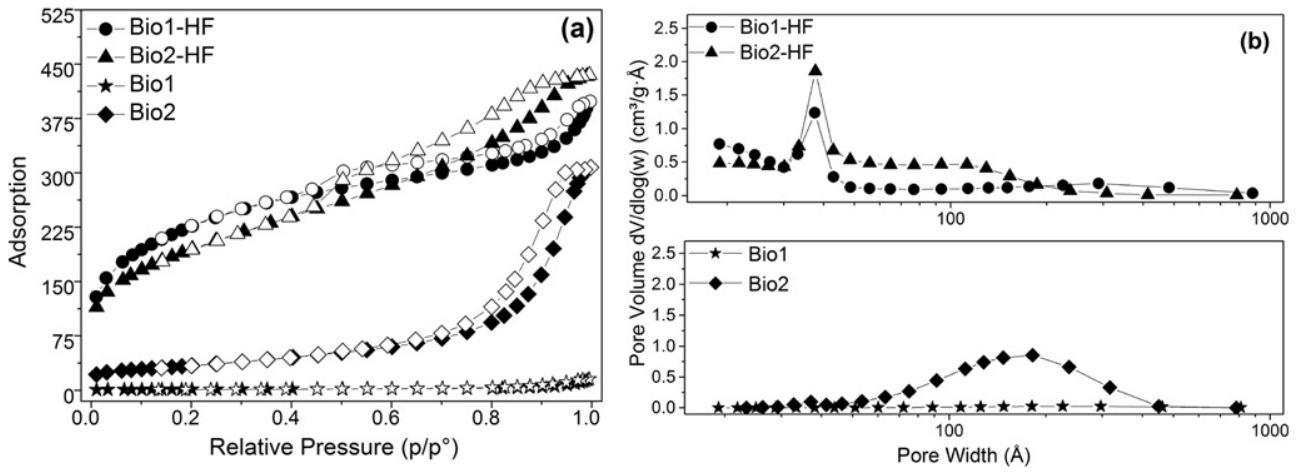

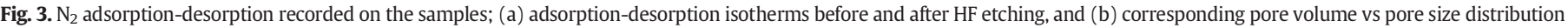

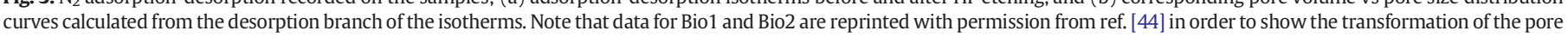
characteristics after HF attack. 

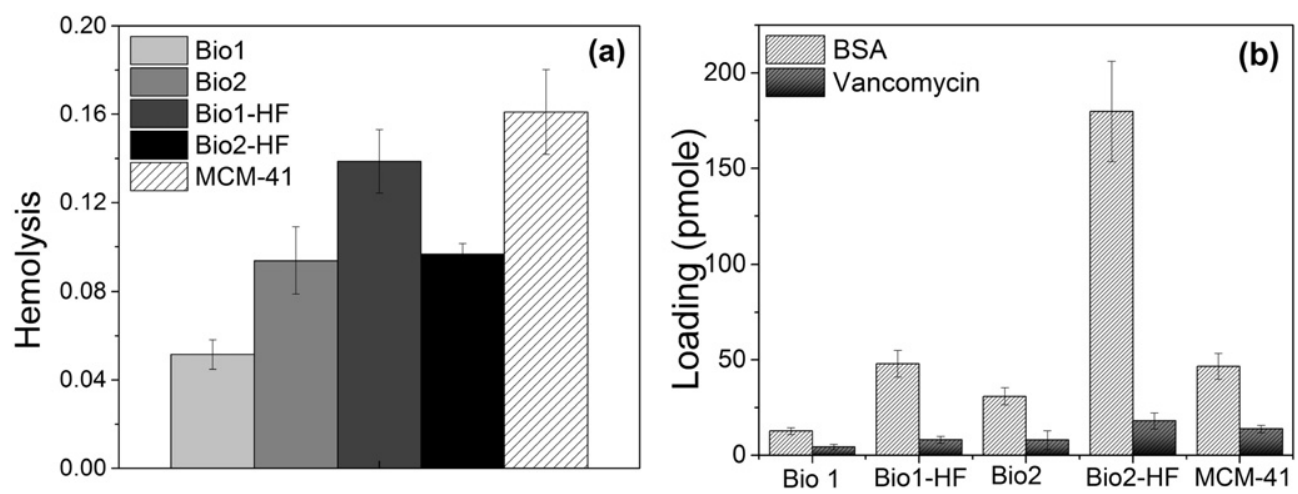

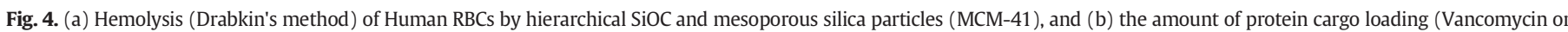
BSA) to each particle was given as pmole.

to BSA release. Similar to MCM-41, all samples investigated (Bio1 and Bio2 with or without HF-etching) have shown comparable release profiles with initial fast release followed by a slower release step; see Fig. 5(b). Among all samples, however, HF treated samples behaved differently showing fewer release of vancomycin after initial fast release stage than that of MCM-41 (and also around same amount less compared to BSA molecules).

It was recently shown that the pore size is an important parameter in determining the drug release kinetics from mesoporous silica particles [58]. However, not only pore size but also molecule chemistry and size (weight) of the used cargo should be critical (different end groups from these cargo molecules may exert additional interactions). Due probably to this mutual interaction between vancomycin molecules and hierarchical porosity and surface chemistry provided in HF treated samples, a longer release profile resulting in about 20\% less release of cargo in $24 \mathrm{~h}$, compared to MCM-41, was observed. This is a significant finding since with such hierarchical porosity materials; diverse cargo molecules having different release kinetics could be delivered at the same time.

Dual/multi cargo loading related works recently seen in the literature $[33,34,59]$, only focused on the utilization of mesoporous nanoparticles (the used MSN particles are mostly having gradient porosity in meso-scale but they do not have hierarchical porosity by definition). Hierarchically porous materials such as SiOC particles produced in the present work, were never used before and could perhaps be more functional in multi drug delivery therapies. Another important point should also be underlined: in the reported studies for dual cargo release, similar sized cargo molecules were utilized. For example, Song et al. [59] reported dual delivery of fluorescein and rhodamine B (MW of 332.3 and $479 \mathrm{Da}$, respectively). Li et al. [33] used silica nanoparticles for codelivery of doxorubicine (MW of $543.5 \mathrm{Da}$ ) and and cisplatin
(300 Da). Fluorescein molecules are documented to be oblate ellipsoid with approximated volume of $0.4 \mathrm{~nm}^{3}$, other molecules with comparable MWs are expected to be in the similar size range [60].

In order to show the capabilities of hierarchically porous SiOC particles, fluorescein and BSA molecules were used as model cargos. Different than those mentioned studies, we chose extreme molecular sized cargo molecules in order to demonstrate capabilities of hierarchically porous materials for co-delivery of molecules having diverse sizes. In dual release studies, the cargo vancomycin was replaced by fluorescein for easy and sensitive detection properties. Fluorescein $\left(\sim 0.4 \mathrm{~nm}^{3}\right.$ \& $332.3 \mathrm{Da}$ ), selected as a model for small sized drugs, is a water soluble molecule with a comparable molecular weight to vancomycin, but much smaller than BSA ( $\left.22.4 \mathrm{~nm}^{3} \& 66,463 \mathrm{Da}\right)$, and frequently used in cargo release experiments with silica particles [61,62].

Bio2-HF and MCM-41 were loaded both with fluorescein and BSA and the release kinetics of each molecule was monitored (Fig. 6(a\&b)). MCM-41 exhibited highly different release kinetics for fluorescein when it is loaded together with BSA (Fig. 7(a)). This can probably be explained by the hindering effect of BSA and thus entrapment of small fluorescein molecules in the micro/mesopores until BSA molecules were detached from the surface of the particles. Therefore, a lagging time in release of fluorescein was observed for MCM-41. In a different manner, the release kinetics of fluorescein and BSA was not affected much when they are loaded together in the hierarchical pores of the Bio2-HF. This allowed release of both large and small cargo molecules at the same time can be beneficial in some combination therapy applications. This explanation is not conclusive in demonstrating differential distribution of the drugs in hierarchical pores, and more studies are needed to elucidate the real mechanism.

The release capabilities of mesoporous silica and SiOC particles were tested in the presence of bacteria, S. aureus. Vancomycin-loaded
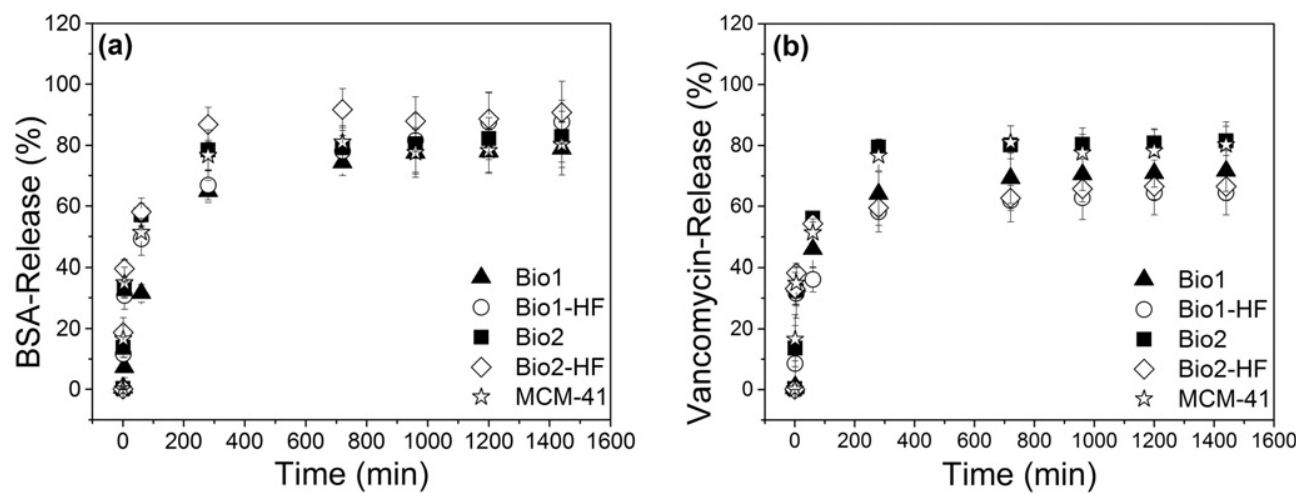

Fig. 5. Release curve of (a) BSA, and (b) vancomycin loaded nanoparticles in PBS buffer. 

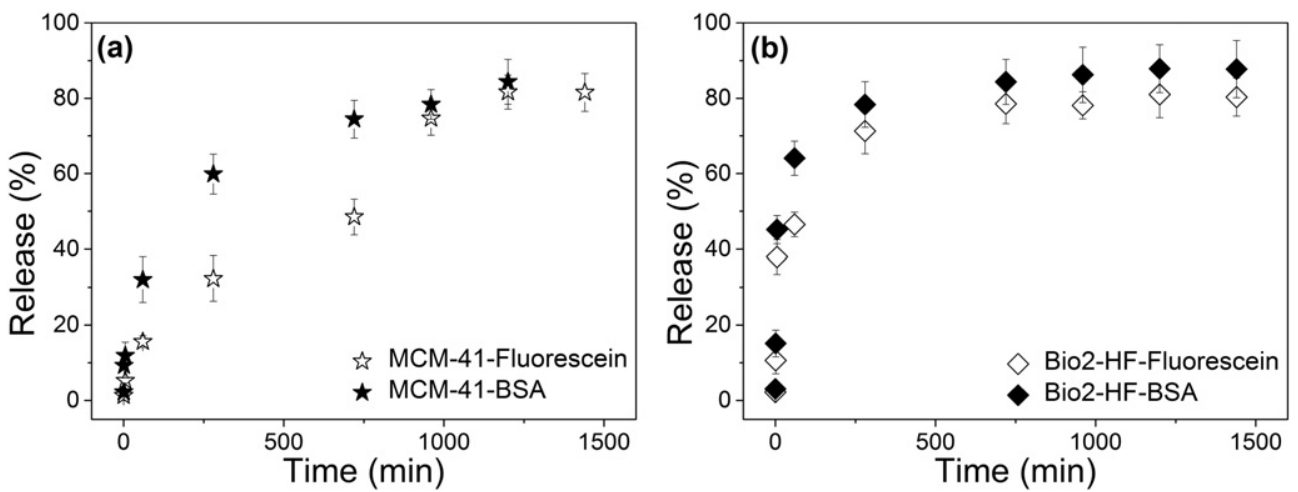

Fig. 6. The release profiles of (a) MCM-41, and (b) Bio2-HF when they are loaded with both fluorescein and BSA molecules simultaneously.

particles were added to $10^{3}$ colony forming units (CFU) bacteria mixtures and the viability of the bacteria was monitored by culture method. Bio2-HF and MCM-41 control particles sample were selected to be used in this time-kill experiments since Bio2-HF demonstrated comparable loading capacity with MCM-41 (Fig. 4(b)). Thus, any difference between two particles would indicate the efficiency of the drug release in killing S. aureus. Time-kill curves were used to study the time course of antimicrobial effects [63].

Fig. 7 shows the effect of 1 MIC concentration of vancomycin-loaded MCM-41 or Bio2-HF and the change in density of live bacteria as a function of time. MIC values were determined according to the loaded concentration of vancomycin [39]. As can be seen in Fig. 7, vancomycinloaded particles can be considered an effective therapeutic strategy against $S$. aureus, by inhibiting bacterial growth since the growth curve started to level off after $2 \mathrm{~h}$. A strong and prolonged bactericidal activity from 2 to $24 \mathrm{~h}$ was observed as compared to control experiment without antibiotic application. Furthermore, the growth curve of S. aureus in presence of vancomycin-loaded Bio2-HF was inhibited slightly better compared to vancomycin-loaded MCM-41 (Fig. 7).

Time-kill results clearly demonstrated the bactericidal efficacy of SiOC loaded with antibiotics and the effect was comparable to mesoporous silica particles. The results also show a slightly better bactericidal activity with vancomycin-loaded Bio2-HF compared to MCM-41. Comparing these two types of tested particles (MCM-41 and Bio2-HF) for effective inhibition of $\mathrm{S}$. aureus growth, use of SiOC as controlled release agents provides alternative opportunities. Due probably to the

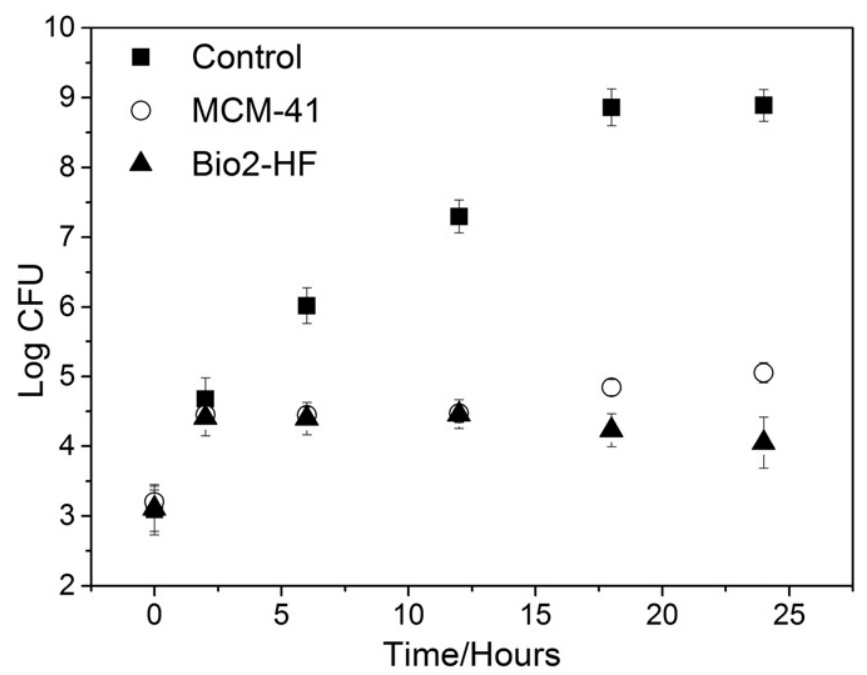

Fig. 7. Time-kill curves for $S$. aureus cultures after treating with vancomycin-loaded MCM41 or Bio2-HF at 1 MIC concentration. Control refers to growth curve without any vancomycin and MCM-41 or Bio2-HF refers to growth curves after addition of vancomycin-loaded particles containing one MIC concentration. hierarchical porosity in Bio2-HF, the release behavior is different than MCM-41 as shown in Fig. 7. From this perspective, succeeding quest exists: (i) for the design of PDC (both etched \& un-etched state) based hierarchical porosity, drug release systems loaded with multiple cargoes, and (ii) for evaluation of their in-vitro properties (adsorption, timekill, etc.).

\section{Conclusions}

Hierarchically porous SiOC samples were made by using 'molecular spacer' templated polymer followed by pyrolysis and hydrofluoric acid etching. The samples showed high specific surface area (SSA) reaching $774 \mathrm{~m}^{2} / \mathrm{g}$ and multi-modal porosity. Such SSA samples were tested for hemolytic behavior, then loading capabilities were measured with two different polypeptides, vancomycin ( $\mathrm{MW}=1449 \mathrm{Da}$ ) and Bovine Serum Albumin (BSA) (MW $=66,463 \mathrm{Da}$ ) having different molecular sizes, and hence for their encapsulation efficiency, release kinetics and biocompatibility. All SiOC samples showed lower hemolysis than that of the commercially available mesoporous silica (MCM-41). The loading capacity studies revealed that one of the carbon-rich SiOC sample (Bio2HF) adsorbed about 3.8 times more BSA and about 1.4 times more vancomycin molecules than MCM-41 particles. Samples showed similar trends in release behavior, and HF treated samples demonstrated $\sim 60 \%$ release, while MCM- 41 was at $~ 80 \%$ after $24 \mathrm{~h}$. Simultaneous multi cargo loading and release capacity tests done by both fluorescein and BSA, and the release kinetics of each molecule was monitored. While a lagging time in release of fluorescein was observed for MCM41 , not much change was observed when such molecules were loaded together in the hierarchical pores of the Bio2-HF, demonstrating for the first time the release of both large and small cargo molecules. Time-kill (for effective inhibition of $S$. aureus growth) tests showed that vancomycin-loaded Bio2-HF sample performed better than vancomycin-loaded MCM-41 in improving bactericidal activity. This study opens new prospects and demonstrates the potential for tailoring custom-made drug (single \& multi cargo) delivery components with suitable hierarchical porosity.

\section{References}

[1] I.Y. Guzman, Certain principles of formation of porous ceramic structures. Properties and applications (a review), Glas. Ceram. 60 (9-10) (2003) 280-283.

2] A.R. Studart, U.T. Gonzenbach, E. Tervoort, L.J. Gauckler, Processing routes to macroporous ceramics: a review, J. Am. Ceram. Soc. 89 (6) (2006) 1771-1789.

[3] P. Colombo, C. Vakifahmetoglu, S. Costacurta, Fabrication of ceramic components with hierarchical porosity, J. Mater. Sci. 45 (20) (2010) 5425-5455.

[4] J. Luyten, S. Mullens, I. Thijs, Designing with pores-synthesis and applications, KONA powder and particle, Journal 28 (2010) 131-142.

[5] C. Vakifahmetoglu, D. Zeydanli, P. Colombo, Porous polymer derived ceramics, Mater. Sci. Eng. R. 106 (2016) 1-30.

[6] Z.Y. Yuan, T.Z. Ren, B.L. Su, $\mathrm{CO}_{2}$-induced micro-construction of hierarchical strings of mesoporous silica spheroids, Chem. Phys. Lett. 383 (3) (2004) 348-352.

[7] P.J. Kooyman, Synthesis strategies for the preparation of mixed micro-mesoporous materials, Stud. Surf. Sci. Catal. 174 (2008) 91-96. 
[8] C. Huiyong, X. Hongxia, C. Xianying, Q. Yu, Experimental and molecular simulation studies of a ZSM-5-MCM-41 micro-mesoporous molecular sieve, Microporous Mesoporous Mater. 118 (1-3) (2009) 396-402.

[9] K. Li, Ceramic Membranes for Separation and Reaction, Wiley, Chichester, England, 2007.

[10] F. Schüth, Endo- and exotemplating to create high-surface-area inorganic materials, Angew. Chem. Int. Ed. 42 (31) (2003) 3604-3622.

[11] G.J.d.A.A. Soler-Illia, C. Sanchez, B. Lebeau, J. Patarin, Chemical strategies to design textured materials: from microporous and mesoporous oxides to nanonetworks and hierarchical structures, Chem. Rev. 102 (11) (2002) 4093-4138.

[12] V. Presser, S.-H. Yeon, C. Vakifahmetoglu, C.A. Howell, S.R. Sandeman, P. Colombo, S. Mikhalovsky, Y. Gogotsi, Hierarchical porous carbide-derived carbons for the removal of cytokines from blood plasma, Adv. Healthc. Mater. 1 (6) (2012) 796-800.

[13] E. Zera, W. Nickel, G.P. Hao, L. Vanzetti, S. Kaskel, G. Soraru, Nitrogen doped carbide derived carbon aerogels by chlorine etching of a SiCN aerogel, J. Mater. Chem. A 4 (12) (2016) 4525-4533.

[14] P. Colombo, L. Biasetto, E. Bernardo, S. Costacurta, C. Vakifahmetoglu, R. PeñaAlonso, G.D. Sorarù, E. Pippel, J. Woltersdorf, Hierarchical porosity ceramic components from preceramic polymers, in: M. Brito, E. Darrel, W.M. Kriven (Eds.), 31st International Conference on Advanced Ceramics and Composites, Wiley, Daytona Beach, Florida 2007, pp. 3-11.

[15] C. Vakifahmetoglu, P. Colombo, A. Pauletti, C.F. Martin, F. Babonneau, SiOC ceramic monoliths with hierarchical porosity, Int. J. Appl. Ceram. Technol. 7 (4) (2010) 528-535.

[16] S.-H. Yeon, P. Reddington, Y. Gogotsi, J.E. Fischer, C. Vakifahmetoglu, P. Colombo, Carbide-derived-carbons with hierarchical porosity from a preceramic polymer, Carbon 48 (1) (2010) 201-210.

[17] Z.C. Eckel, C. Zhou, J.H. Martin, A.J. Jacobsen, W.B. Carter, T.A. Schaedler, Additive manufacturing of polymer-derived ceramics, Science 351 (6268) (2016) 58-62.

[18] A. Tolosa, B. Krüner, N. Jackel, M. Aslan, C. Vakifahmetoglu, V. Presser, Electrospinning and electrospraying of silicon oxycarbide-derived nanoporous carbon for supercapacitor electrodes, J. Power Sources 313 (2016) 178-188.

[19] V.L. Nguyen, E. Zera, A. Perolo, R. Campostrini, W. Li, G.D. Sorarù, Synthesis and characterization of polymer-derived SiCN aerogel, J. Eur. Ceram. Soc. 35 (12) (2015) 3295-3302.

[20] D. Assefa, E. Zera, R. Campostrini, G.D. Soraru, C. Vakifahmetoglu, Polymer-derived SiOC aerogel with hierarchical porosity through HF etching, Ceram. Int. 42 (10) (2016) 11805-11809.

[21] M. Vallet-Regí, M.M. Garcia, M. Colilla, Biomedical Applications of Mesoporous Ceramics: Drug Delivery, Smart Materials and Bone Tissue Engineering, CRC Press, 2012.

[22] M.M. De Villiers, P. Aramwit, G.S. Kwon, Nanotechnology in Drug Delivery, Springer Science \& Business Media, 2008.

[23] S. Kwon, R.K. Singh, R.A. Perez, E.A.A. Neel, H.-W. Kim, W. Chrzanowski, Silica-based mesoporous nanoparticles for controlled drug delivery, J. Tissue Eng. 4 (2013) (2041731413503357).

[24] Q. He, J. Shi, Mesoporous silica nanoparticle based nano drug delivery systems: synthesis, controlled drug release and delivery, pharmacokinetics and biocompatibility, J. Mater. Chem. 21 (16) (2011) 5845-5855.

[25] A.B.D. Nandiyanto, S.-G. Kim, F. Iskandar, K. Okuyama, Synthesis of spherical mesoporous silica nanoparticles with nanometer-size controllable pores and outer diameters, Microporous Mesoporous Mater. 120 (3) (2009) 447-453.

[26] C.C. Lechner, C.F.W. Becker, Silaffins in Silica Biomineralization and Biomimetic Silica Precipitation, Mar. Drugs 13 (8) (2015) 5297-5333.

[27] E. Sayed, R. Haj-Ahmad, K. Ruparelia, M.S. Arshad, M.-W. Chang, Z. Ahmad, Porous inorganic drug delivery systems-a review, AAPS PharmSciTech 18 (5) (2017) $1507-1525$.

[28] A. Tamayo, M.A. Mazo, M.D. Veiga, R. Ruiz-Caro, F. Notario-Pérez, J. Rubio, Drug kinetics release from Eudragit - Tenofovir@SiOC tablets, Mater. Sci. Eng. C 75 (2017) 1097-1105.

[29] A. Tamayo, M.A. Mazo, R. Ruiz-Caro, A. Martín-Illana, L.M. Bedoya, M.D. VeigaOchoa, J. Rubio, Mesoporous silicon oxycarbide materials for controlled drug delivery systems, Chem. Eng. J. 280 (2015) 165-174.

[30] R. Zhuo, P. Colombo, C. Pantano, E.A. Vogler, Silicon oxycarbide glasses for bloodcontact applications, Acta Biomater. 1 (5) (2005) 583-589.

[31] J. Grossenbacher, M.R. Gullo, F. Dalcanale, G. Blugan, J. Kuebler, S. Lecaudé, H. Tevaearai Stahel, J. Brugger, Cytotoxicity evaluation of polymer-derived ceramics for pacemaker electrode applications, J. Biomed. Mater. Res. A 103 (11) (2015) 3625-3632.

[32] G.M. Renlund, S. Prochazka, R.H. Doremus, Silicon oxycarbide glasses: part II. Structure and properties, J. Mater. Res. 6 (12) (1991) 2723-2734.

[33] H. Li, H. Yu, C. Zhu, J. Hu, M. Du, F. Zhang, D. Yang, Cisplatin and doxorubicin dualloaded mesoporous silica nanoparticles for controlled drug delivery, RSC Adv. 6 (96) (2016) 94160-94169.

[34] X. Chen, Z. Liu, Dual responsive mesoporous silica nanoparticles for targeted codelivery of hydrophobic and hydrophilic anticancer drugs to tumor cells, J. Mater. Chem. B 4 (25) (2016) 4382-4388.

[35] M. Wu, Y. Chen, L. Zhang, X. Li, X. Cai, Y. Du, L. Zhang, J. Shi, A salt-assisted acid etching strategy for hollow mesoporous silica/organosilica for $\mathrm{pH}$-responsive drug and gene co-delivery, J. Mater. Chem. B 3 (5) (2015) 766-775.
[36] C. Vakifahmetoglu, M. Buldu, A. Karakuscu, A. Ponzoni, D. Assefa, G.D. Soraru, High surface area carbonous components from emulsion derived SiOC and their gas sensing behavior, J. Eur. Ceram. Soc. 35 (16) (2015) 4447-4452.

[37] D.L. Drabkin, J.H. Austin, Spectrophotometric studies II. Preparations from washed blood cells; nitric oxide hemoglobin and sulfhemoglobin, J. Biol. Chem. 112 (1) (1935) 51-65.

[38] J.M. Swenson, K.F. Anderson, D.R. Lonsway, A. Thompson, S.K. McAllister, B.M Limbago, R.B. Carey, F.C. Tenover, J.B. Patel, Accuracy of commercial and reference susceptibility testing methods for detecting vancomycin-intermediate Staphylococcus aureus, J. Clin. Microbiol. 47 (7) (2009) 2013-2017.

[39] M. Kavruk, O. Celikbicak, V.C. Ozalp, B.A. Borsa, F.J. Hernandez, G. Bayramoglu, B. Salih, M.Y. Arica, Antibiotic loaded nanocapsules functionalized with aptamer gates for targeted destruction of pathogens, Chem. Commun. 51 (40) (2015) 8492-8495.

[40] M.M. Bradford, A rapid and sensitive method for the quantitation of microgram quantities of protein utilizing the principle of protein-dye binding, Anal. Biochem. 72 (1) (1976) 248-254.

[41] Y. Blum, G.D. Sorarù, A.P. Ramaswamy, D. Hui, S.M. Carturan, Controlled mesoporosity in SiOC via chemically bonded polymeric "spacers", J. Am. Ceram. Soc. 96 (9) (2013) 2785-2792.

[42] S. Dirè, V. Tagliazucca, L. Salvadori, G.D. Sorarù, Preparation of dense and porous silicon Oxycarbide submicrometer-sized spheres using a modified Stöber process, J. Am. Ceram. Soc. 94 (11) (2011) 3819-3824.

[43] K. Lu, Porous and high surface area silicon oxycarbide-based materials-a review Mater. Sci. Eng. R. 97 (2015) 23-49.

[44] C. Vakifahmetoglu, D. Zeydanli, M.D.d.M. Innocentini, F.d.S. Ribeiro, P.R.O. Lasso, G.D Soraru, Gradient-hierarchic-aligned porosity SiOC ceramics, Sci. Rep. 7 (2017) 41049.

[45] J. Wu, Y. Li, L. Chen, Z. Zhang, D. Wang, C. Xu, Simple fabrication of micro/nanoporous SiOC foam from polysiloxane, J. Mater. Chem. 22 (14) (2012) 6542-6545.

[46] C.T. Kresge, M.E. Leonowicz, W.J. Roth, J.C. Vartuli, J.S. Beck, Ordered mesoporous molecular sieves synthesized by a liquid-crystal template mechanism, Nature 359 (6397) (1992) 710-712.

[47] A. Zukal, M. Thommes, J. Cejka, Synthesis of highly ordered MCM-41 silica with spherical particles, Microporous Mesoporous Mater. 104 (1-3) (2007) 52-58.

[48] A. Saha, R. Raj, D.L. Williamson, A model for the nanodomains in polymer-derived SiCO, J. Am. Ceram. Soc. 89 (2006) 2188-2195.

[49] H. Wu, J. Yang, H. Chen, F. Pan, Revealing the nanodomain structure of silicon oxycarbide via preferential etching and pore analysis, Funct. Mater. Lett. 09 (03) (2016) 1650043.

[50] R. Peña-Alonso, G. Mariotto, C. Gervais, F. Babonneau, G.D. Soraru, New insights on the high-temperature nanostructure evolution of SiOC and B-doped SiBOC polymer-derived glasses, Chem. Mater. 19 (23) (2007) 5694-5702.

[51] G.D. Sorarù, R. Pena-Alonso, H.-J. Kleebe, The effect of annealing at $1400{ }^{\circ} \mathrm{C}$ on the structural evolution of porous C-rich silicon (boron)oxycarbide glass, J. Eur. Ceram. Soc. 32 (8) (2012) 1751-1757.

[52] I.I. Slowing, C.-W. Wu, J.L. Vivero-Escoto, V.S.Y. Lin, Mesoporous silica nanoparticles for reducing hemolytic activity towards mammalian red blood cells, Small 5 (1) (2009) 57-62.

[53] A. Tamayo, R. Peña-Alonso, J. Rubio, R. Raj, G.D. Sorarù, J.L. Oteo, Surface energy of sol gel-derived silicon Oxycarbide glasses, J. Am. Ceram. Soc. 94 (12) (2011) 4523-4533.

[54] L. Cui, A. Iwamoto, J.-Q. Lian, H.-M. Neoh, T. Maruyama, Y. Horikawa, K. Hiramatsu, Novel mechanism of antibiotic resistance originating in vancomycin-intermediate Staphylococcus aureus, Antimicrob. Agents Chemother. 50 (2) (2006) 428-438.

[55] S.P. Maddala, G. Mastroianni, D. Velluto, A.C. Sullivan, Intracellular delivery of BSA by phosphonate@silica nanoparticles, J. Mater. Chem. B 3 (29) (2015) 6057-6070.

[56] L.A. Perelman, C. Pacholski, Y.Y. Li, M.S. VanNieuwenhze, M.J. Sailor, pH-triggered release of vancomycin from protein-capped porous silicon films, Nanomedicine 3 (1) (2008) 31-43.

[57] V.C. Özalp, T. Schäfer, Aptamer-based switchable nanovalves for stimuli-responsive drug delivery, Chem. Eur. J. 17 (36) (2011) 9893-9896.

[58] E.L. Pastor, E. Reguera-Nuñez, E. Matveeva, M. Garcia-Fuentes, Pore size is a critical parameter for obtaining sustained protein release from electrochemically synthesized mesoporous silicon microparticles, Peer J. 3 (2015), e1277.

[59] B. Song, C. Wu, J. Chang, Dual drug release from electrospun poly(lactic-co-glycolic acid)/mesoporous silica nanoparticles composite mats with distinct release profiles, Acta Biomater. 8 (5) (2012) 1901-1907.

[60] Y. Pu, W. Wang, R.B. Dorshow, R.R. Alfano, Picosecond Polarization Spectroscopy of Fluorescein Attached to Different Molecular Volume Polymer Influenced by Rotational Motion, 2012825818.

[61] V.C. Özalp, A. Pinto, E. Nikulina, A. Chuvilin, T. Schäfer, In situ monitoring of DNAAptavalve gating function on mesoporous silica nanoparticles, Part. Part. Syst. Charact. 31 (1) (2014) 161-167.

[62] F. Sancenón, L. Pascual, M. Oroval, E. Aznar, R. Martínez-Máñez, Gated silica mesoporous materials in sensing applications, Chem. Open 4 (4) (2015) 418-437.

[63] T.J. Dilworth, J. Sliwinski, K. Ryan, M. Dodd, R.-C. Mercier, Evaluation of vancomycin in combination with piperacillin-tazobactam or oxacillin against clinical methicillinresistant Staphylococcus aureus isolates and vancomycin-intermediate S. aureus isolates in vitro, Antimicrob. Agents Chemother. 58 (2) (2014) 1028-1033. 